\title{
Pistes de recherche pour une étude sur les procureurs généraux syndics : entre histoire individuelle et histoire institutionnelle
}

New perspectives for research on the "procureurs généraux syndics »: between the history of individuals and the history of institutions

\section{Gaid Andro}

\section{(2) OpenEdition Journals}

\section{Édition électronique}

URL : https://journals.openedition.org/ahrf/11632

DOI : 10.4000/ahrf.11632

ISSN : 1952-403X

\section{Éditeur :}

Armand Colin, Société des études robespierristes

Édition imprimée

Date de publication : 1 juin 2010

Pagination : $3-25$

ISBN : 978-2-200-92632-8

ISSN : 0003-4436

\section{Référence électronique}

Gaid Andro, «Pistes de recherche pour une étude sur les procureurs généraux syndics : entre histoire individuelle et histoire institutionnelle », Annales historiques de la Révolution française [En ligne], 360 | avril-juin 2010, mis en ligne le 01 juin 2013, consulté le 23 avril 2022. URL : http:// journals.openedition.org/ahrf/11632 ; DOI : https://doi.org/10.4000/ahrf.11632 


\title{
PISTES DE RECHERCHE POUR UNE ÉTUDE SUR LES PROCUREURS GÉNÉRAUX SYNDICS : ENTRE HISTOIRE INDIVIDUELLE ET HISTOIRE INSTITUTIONNELLE
}

Gaid ANDRO

\begin{abstract}
Lorsque les constituants établissent la nouvelle pyramide administrative française dans les premiers mois de la Révolution, ils confient la mise en application de la loi au procureur général syndic du département. Élu par les assemblées électorales départementales, le procureur général syndic est théoriquement la synthèse institutionnelle de deux objectifs difficilement conciliables: l'exigence démocratique locale et le contrôle du territoire par l'exécutif central. Cette tension propre à la fonction laisse subsister un certain flou quant aux prérogatives réelles de ces hommes. Selon les départements, il apparaît comme un administrateur secondaire sans grand pouvoir de décision ou au contraire comme un personnage central de la construction identitaire départementale. Une unité apparaît cependant lors de l'analyse de leur recrutement et de leurs carrières. L'approche prosopographique permet ainsi de replacer cette institution dans la problématique plus large de la construction de l'État à travers la formation d'une nouvelle élite administrative.
\end{abstract}

Mots-clés: procureur général syndic, prosopographie, administration départementale, savoir-faire administratif, notabilité.

L'historiographie a longtemps fait la part belle àl'histoire politique et institutionnelle de la Révolution française. Les connaissances en ce domaine sont précises etles nombreuxchantiers de recherche, alimentés localement par les travaux universitaires, participent à peindre de plusen plus précisémentla réalitédu processus révolutionnaireentermedevécu et de pratique politique.L'histoire delaconstruction del'État suitainsison 
proprerythme,perméableauxmodesetauxcourantshistoriographiques, laissant parfois dansl'ombre des institutions moins attractives auprès des chercheurs. C'est en partie le cas des procureurs généraux syndics mis en place dès les premiers mois de la Révolution pour incarner le pouvoir exécutifdanslesdépartements.Cetteinstitutionéphémère,suppriméeen I'an II, reste encore peu connue alors qu'elle concrétise une partie des ambitions révolutionnaires de 1789. Afin de redonnerune placeà ces personnalités politiques, il est nécessaire d'envisager l'institution à travers deux axes.

Il convient tout d'abord de dépasser l'échec institutionnel des procureurs générauxsyndicsafin de sortird'une perception téléologiquequi réduit leur existence à une simple phase de transition. Si l'on se replace dans l'intentionnalité des constituants de 1789, les procureurs généraux syndicsnesontpasplusunetransitionquelamonarchieconstitutionnelle elle-même.Dansunsecondtemps, cetteinstitutiontrouveunerésonance dans ses répercussions à long terme. Elle pose des questions propres aux acteurs politiques de la Révolution en interrogeant sur le devenir d'une nouvellegénérationd'hommesformésdanslecreusetdel'administration départementale'.

Le procureur général syndic :

une séparation des pouvoirs au sein du département

Après l'été 1789 , les constituants ont la responsabilité de fonder un ordre social nouveau. Cette société réinventée doit reposer sur une administration associant la légitimité électorale et l'autorité institutionnelle, deux composantes inscrites dans un territoire naissant :le département. Aucoursdesinterminables débatsentreconstituants, laquestiondelaprésence concrète de l'exécutif dans l'ensemble du pays se pose avec force. Lamonarchieconstitutionnelledoitpouvoirsedoterd'unreprésentantdu pouvoircentralauniveaudépartemental.Leshommesde1789rejoignent ici une problématique constante de la construction de l'État, une problé-

(1) Notre article présente ici les pistes de recherches établies lors de la première année de thèse.Cetravail,amorcéenoctobre2006apourambitiondemeneruneétudeapprofondiesurlesprocureurs généraux syndics français entre 1790 et 1793 . Notre recherche se conçoit dans une perspective large au niveau géographique (l'ensemble du territoire français) et chronologique (la logique prosopographique inclut en effet les années précédant la Révolution et le xIx ${ }^{e}$ siècle). Elle s'inscrit dans la continuité des importants travaux de Gilbert SCHNEIDER, « Les procureurs généraux syndics sous la Révolution. Réflexions etéléments pour une enquête prosopographique ", mémoire de DEA, sous la direction de Claude Mazauric, Université de Rouen, 1996. 
matiquelargement traitée dans l'historiographie politiqueet révolutionnaire ${ }^{2}$. Cependant, ces débats ont lieu alors que la priorité collective est de mettre à bas l'arbitraire royal et de limiter les pouvoirs du monarque. L'arbitraire se combat à toutes les échelles et, dans le cadre provincial, lesconstituants sontmajoritairementcritiquesenverslesprérogatives de l'intendant qu'ils dénoncent comme l'incarnation locale du despotisme monarchique. Les nouveaux objectifs sont donc posés en ces termes : le pouvoir central doit avoir une représentation concrète, mais limitée, au sein del'administration départementale. Aucunagent directnedoitpouvoirs'arroger detroplarges prérogatives au niveau du département. Ainsi naîtleprocureurgénéral syndic, unenouveautéinstitutionnelleélaborée comme un contre modèle de l'Ancien Régime.

Ce personnage est en effet construit en négatif, c'est-à-dire à partir des craintes des constituants. L'institution tout entière est limitée par la peur de ce qu'elle pourrait devenir, par les dangers potentiels de sa place danslapyramideadministrative.Leprocureurgénéral syndicestun représentant du roi, il est sous son autorité par l'intermédiaire des ministres. Il est chargé de transmettre les lois au directoire de département et aux procureurs syndics des districts selon les règles de la hiérarchie administrative. Il rappelle aux membres de l'administration les principes de la loi lors de l'exercice particulier du réquisitoire. Chaque décision administrative prise par le département répond à la fois à une problématique locale et à une orientation légale nationale. En maîtrisant ce double objectif, le procureurgénéralsyndicseplaceaucentredufonctionnementdel'appareil exécutif ${ }^{3}$. Dans le même temps, il ne peut être considéré comme un pivot administratif, c'est-à-dire comme une strate de l'administration; il reste accolé au directoire de département. Il est donc une incarnation de l'exécutif au cœur de l'administration du département et non au sein du territoire départemental.Cequi, en soi, le différencie fondamentalement desautresincarnationsdel'exécutifreconnuesparl'historiographietelles

(2) Nous faisons ici notamment référence au récent ouvrage de Michel BIARD, Les lilliputiens de la centralisation. Des intendants aux préfets, les hésitations d'un « modèle » français, Seyssel, Champ Vallon, 2007. Cet ouvrage développe une analyse critique de la thèse de Tocqueville surla continuitéadministrative de la centralisation dans la construction del'État français. La question étant alors de réfléchir à la place de la première constitution de 1791 dans ce processus de centralisation en suivant notamment Alphonse Aulard et son idée de « décentralisation de circonstance ».

(3) Le réquisitoire est un point central de la fonction du procureur général syndic. Avant chaque délibération, il a la parole et expose au directoire un état des problèmes, les principes de la loi et les décisions à prendre selon lui. Le réquisitoire illustre chez le procureur une capacité oratoire ainsi qu'une maîtrise globale des différents domaines de l'administration. 
quel'intendant ou le préfet de la République. En le « décalant » de la pyramide administrative, les constituants limitent de fait son pouvoir dans la prise de décision administrative. Ils concrétisent ainsi la séparation nette entre pouvoir exécutif et pouvoir législatif. En héritiers de la philosophie du XVIII ${ }^{e}$ siècle et de la pensée politique de Montesquieu, ils dissocient ceux qui écrivent la loi de celui qui doit veiller à son exécution. Or les hommes de 1789ont profondémentminimiséle rôledel'exécutifparrapport au législatif ${ }^{4}$. Le fondement de la souveraineté du peuple reposant sur l'aptitude à créer la loi plus que sur l'obligation à la faire appliquer. L'institution des procureurs syndics et plus largement le système administratif de 1790 doivent donc se penser dans la perspective large de la pensée politique du xvIII siècle. Les procureurs généraux syndics sont institués le 10 décembre 1789 à l'Assemblée constituante en ces termes: «Enchaqueadministration dedépartementilyauraun procureurgénéral syndicetdanschaqueadministration dedistrictilyaura un procureursyndic. Le procureur général syndic et les procureurs syndics seront quatre ansmisen placeet pourrontêtre continués pourautresquatreans parune nouvelle élection; mais après huit ans d'exercice, ils ne pourront plus être continués ni même réélus qu'après un intervalle de deux ans au moins ». Ainsi, même si le procureur général syndic possède, comme l'ensemble del'administration, la légitimité du suffrage, la loilimite toute installation dans la charge et par là même toute personnalisation de l'exécutif dans le département. Le suffrage est surtout ici un moyen de court-circuiter la tutelle de l'exécutif central sur l'administration et non une délégation de la souveraineté nationale. En effet, seuls les députés, et par eux le pouvoir législatif, ont un caractère représentatif en tant qu'incarnation de la souveraineté du peuple.

Dès 1789 les bornes sont donc explicitement posées pour limiter les prérogatives de l'administration et a fortiori du procureur général syndic. Car si l'institution collégiale du directoire semble prémunir contre tous les abus de pouvoir, la place de procureur en tant qu'intermédiaire individuel reste potentiellement dangereuse. Les débats de février 1791

(4) Michel Verpeaux, La naissance du pouvoir réglementaire (1789-1799), Paris, PUF, 1991. L'auteur souligne ici les conceptions philosophiques des constituants de 1789 et la double influence de Montesquieu et de Rousseau dansla miseen place dela premièreadministration révolutionnaire. Alors que Montesquieu motive la séparation des pouvoirs, Rousseau initie la domination du législatif sur l'exécutif. Pour les hommes de 1789, le seul pouvoir souverain est celui de l'élaboration des lois. Dès lors «l'exécution des lois n'est pas le problème majeur des révolutionnaires de $1789 »$ ( p. 25) et la question de l'agent de l'exécutif au cœur du département répercute sans aucun doute cette inégalité structurelle. 
sur la constitution des listes dejurés dans l'organisation de lajustice criminelletraduisentencoreclairementcettemenace.Alorsquecertains députésproposentqueleslistessoientrédigéesparleprocureurgénéralsyndic, Robespierre prend la parole en ces termes : «Si la liste des jurés devait être dressée par un seul homme, sous le prétexte qu'il agit sous les regards de ses concitoyens, nous devrions aussi confier à un seul homme toutes les fonctions publiques [...]. C'est surtout dans les temps de Révolution et de faction que rien n'est plus dangereux que de mettre entre les mains d'un seul homme deschoixque peut dirigerl'esprit de partialité »5. L'intervention de Robespierre est d'autant plus intéressante qu'elle peut se lireà lalumièredesenseignementsdugouvernementrévolutionnaire.Eneffet, trois ans plus tard, Robespierre est de ceux qui défendent les prérogatives dureprésentantdupeupleenmissionaucœurdesdépartements.Etfinalement, alors que la lutte des factions pèse sur les orientations politiques de laRévolution,lesreprésentantsdupeupleconcentrentlespouvoirsausein de leur territoire de mission assumant alors « l'esprit de partialité » tant craint en 1791. Les circonstances ont changé et les pratiques politiques aussi. En 1789, l'institution du procureur général syndic repose sur une utopie qui n'envisage pas les tensions internes au processus révolutionnaire. Elle disparaît d'ailleurs en frimaire an II, victime de son incapacité structurelle à résister aux conflits au sein de la société française.

La Constitution de 1791 misait sur l'emboîtement administratif, les municipalités étant attachées aux districts, eux-mêmes contrôlés par uneadministrationdépartementalequirendaitdirectementcompteaugouvernement et au corps législatif. C'est uniquement dans cette pyramide administrative « contrôlée » que le procureur général syndic pouvait agir commereprésentantdupouvoircentralsansdélégationdepouvoiretsans rôle de surveillance. Ainsi, c'est sa propre fonction qu'il légitime lorsque le procureur général syndic de la Meuse, Gossin, écrit aux juges de paix dudépartement : Cetteconcorde,cetteharmonieestundesbienfaitsde la Constitution, elle a liéen un faisceau tous les hommes publics pour que le respect de la loi, de la liberté, des propriétés des citoyens trouvent des défenseurs unis par les mêmes principes $»^{6}$. L'institution se fonde donc sur le principe de l'harmonie administrative et de la paix sociale. Un principethéoriquelargementsabordéparlesrapportsdeforceinternesàtoute

(5) Le Moniteur de la Révolution, Bulletin de l'Assemblée nationale, samedi 5 février 1791, p. 311.

(6) $A N, F^{1 a} 423$ Meuse. Lettre du procureur général syndic aux juges de paix du département (9 décembre 1791). 
organisation politique, a fortiori en période révolutionnaire 7 . De plus, le contrôle étroit du citoyen sur les membres de l'administration par le suffrage, toutencantonnantl'État dans ses limites, conduitinévitablementà une fusion entre le politique et l'administratif. En période de troubles, ce quidevaitassurerlecontrôle desautorités constituées aboutitàla désarticulation de la machine administrative ${ }^{8}$. Plus jamais un système politique françaisn'abandonneral'idéedureprésentantdupouvoircentralnommé etenvoyédansles départements.Lesreprésentantsdupeupleenmission et les préfets de la Républiqueachèveront de renvoyer le procureurgénéral syndic au rayon des innovations avortées de l'histoire.

D'ailleurs l'historiographie révolutionnaire a largement minoré cette institution et parfois même ces administrateurs des premiers temps de la Révolution. Dès le xixe siècle Thiers condamne les procureurs généraux syndics en en faisant des " commissaires du gouvernement central chargés de solliciter auprès d'elles [...] mais privés du pouvoir d'agir eux-mêmes $»^{9}$. Or les écrits de Thiers font autorité chez les penseurs du droitconstitutionnel qui, àl'image deThéophile Ducrocq dans les années 1890,condamnentl'impuissancedesprocureursgénérauxfaceàuneadministration départementale seule détentrice du pouvoir d'agir ${ }^{10}$. Michelet renforce cette image d'inefficacité en parlant des débuts dela Révolution comme d'une « anarchie administrative $»^{11}$. Le système doit avouer son

(7) La question se pose notamment de la surveillance des autorités constituées en ellesmêmes.Dansunepériodedetension,lesadministrationsdoiventpouvoirêtrecontrôléesnotamment par les agents de l'exécutif et donc les procureurs généraux, or comme l'écrit Gérard SAUTEL : « Dans les faits, étant proprement les compagnons d'élection des administrateurs, ils manifestèrent peu de vocation à être leurs contrôleurs » ("Centralisation et décentralisation », dans Administration de la France sous la Révolution, Paris, Droz, 1992, p. 45).

(8) Vida Azımı, «Servir la Nation : agents et fonctionnaires publics », dans Administration de la France sous la Révolution, op. cit, p. 73. L'auteur insiste notamment sur le paradoxe de la conception libérale du contrôle du citoyen sur les fonctionnaires qui aboutit finalement au dysfonctionnement de la toute la machine administrative expliquant en partie l'échec de l'institution elle-même.

(9) Adolphe Thiers, Histoire du Consulat et de l'Empire, Paris, Paulin, 1845, tome 1, p149-155.Thiersimposedurablementl'imaged'uneadministrationdépartementale « délibérantperpétuellement et n'agissant jamais » où les procureurs font office de présence inefficace. L'échec des procureurs légitimant le retour à des représentants nommés et à une centralisation plus directe dès I'an II et surtout sous le Consulat.

(10) Théophile Ducroce, Les procureurs syndics de 1790 et les commissaires du directoire exécutif de l'an III à l'an VIII avec I'histoire de l'institution dans le département de la Vienne, Paris, Ernest Leroux, 1892. L'auteur est professeur à la faculté de droit de Paris et doyen honoraire de la faculté de Poitiers. En relayant les écrits de Thiers et en les enseignant, il officialise la réputation de l'institution et lui assure une postérité tout au long du siècle suivant.

(11) Onretrouvel'étatd'« anarchielégale »dénoncéeparTaineà proposdesadministrations locales. L'anarchie désigne ici cette administration de la fin du xVIII siècle où les administrations départementalessontcenséesêtrelescirconscriptionsdel'action del'exécutiftouten restantbridées par le suffrage. 
échec face à la crise multiforme de 1792. Cette théorie s'assure alors une longue postérité, minimisant à long terme l'action des directoires de département et surtout des procureurs, impuissants face au dérèglement de la société. L'anarchie administrative de 1790 trouve encore un écho dans les écrits d'Albert Soboul. L'opportunité est facile pour légitimer le recours aux représentants en mission puis au gouvernement révolutionnaire. Le jugement de Soboul sur le procureur général syndic est cinglant : « II représentait l'intérêt général, il fut en fait le secrétaire des services administratifs ${ }^{12}$. Réduit à la fonction de secrétaire, le procureur général syndic perdait définitivement tout intérêt pour la recherche. Le vide historiographique fut pourtant souligné par Jacques Godechot dès $1951^{13}$. Mais s'il déplore l'absence d'un travail d'ampleur sur les procureurs généraux syndics, c'est surtout une enquête sur l'esprit public qu'il envisage à travers l'étude de l'élection locale. L'institution n'est donc pas envisagéeparGodechotcommeunobjet derechercheà partentièremais biencommeunsignifiant, unaxedelecturedanslevastechantierdesélections révolutionnaires. Plus récemment, Bernard Gainot soulignait dans un article sur les commissaires du Directoire l'abandon des procureurs généraux syndics et de leurs successeurs aux seuls érudits locaux ${ }^{14}$. Le constat a donc été fait, traversant les générations d'historiens et les courants historiographiques.

L'oubli historiographique des procureurs généraux syndics s'explique par la courte existence de l'institution (juin 1790-frimaire an II), par son échec, par la relative absence de ces hommes dans les sources, mais aussi par l'image de conciliation, de modération et donc de « modérantisme » qu'ils ont laissée dans la mémoire collective, dans la mémoire départementaleet dansl'historiographieduxix siècle. Leurmoded'élection au suffrage censitaire par la population la plus aisée et donc la moins « radicale » en a souvent fait des hommes du consensus. Consensusentre lesministresetl'administrationdedépartement,consensusentrelespopulationsdudépartementetlesassembléesparisiennes, consensusentreprovincialisme et loi nationale. Or c'est comme si l'historiographie de l'État

(12) Albert Soвоul, Précis d'histoire de la Révolution française, Paris, Éditions sociales, 1975,p. 161. On ne peut s'empêcher de penser queles procureurs généraux syndicsn'eurent pas leur chancefaceà une historiographielargementfavorableàla dynamiquecentralisatrice dansla logique républicainecommedanslatraditionmarxiste. Repensercetteinstitution peutdoncameneràrepenser les grands courants de l'historiographie révolutionnaire.

(13) Jacques Godechot, Les Institutions de la France sous la Révolution et l'Empire, Paris, PUF, 1951.

(14) Bernard GAINOT, «La provinceau cribledes rapports descommissaires départementaux du Directoire », AHRF, n³30, oct-déc. 2002, p. 143. 
s'étaitpenchéeplusspontanémentsurleconflitque surleconsensus, laissant au bord de la routeces personnagesn'ayant pas euassez de pouvoirs pour avoir commis des excès.

Condamnéeparlesrévolutionnaireseux-mêmes dès 1793, puispar les historiens dès l'aube du xix siècle, l'institution des procureurs généraux syndics méritait sans doute une recherche plus approfondie autour de plusieurs questions distinctes et complémentaires : cette institution fut-elle vraiment " privée d'influence et frappée d'impuissance $»^{15}$ ? et, plus largement, ne fut-elle pas un cadre d'apprentissage du politique et dusavoir-faireadministratifauseind'un systèmeen recherched'unenouvelle classe dirigeante?

Le procureur général syndic :

une simple personnalité protocolaire?

La fonction du procureur général syndic dans l'administration départementale est posée en ces termes par la loi de décembre 1789 : «Les procureurs généraux syndics et les procureurs syndics assisteront à toutes les assemblées d'administration sans voix délibérative; mais nul rapportne sera fait avant leur en donner communication et nulle délibération ne sera prise sur le rapport sans les avoir entendus ». Lors des réunionsdel'administration départementale, leprocureurdoitdoncfaire un réquisitoire avant chaque prise de décision. II rappelle ainsi la loi et amorce le débat mais il ne peut ensuite parler « qu'à la fin des débats pour donner ses conclusions ». Nous sommes alors au cœur même de l'ambiguïté de l'institution, au cœur d'un entre-deux difficilement compréhensible si l'on néglige les circonstances de 1789. Le procureur doit assister à tous les débats, tout doit lui être communiqué et aucune décision ne peut être prise sans l'écouter. Dans le même temps, il ne peut pas voter et ne peut intervenir qu'une fois dans la discussion. Il incarne donc cette doublevolontési difficilement conciliable :lecontrôle du pouvoircentral etlalimitation du pouvoirindividuel.Sonaction sembledonccirconscrite à une simple présence formelle. De plus, les archives départementales laissent peu de place à ses interventions puisque les réquisitoires sont rarement retranscrits dans les registres du directoire et quesa présencese distingue souventàla seule signature au bas des arrêtés, perdue parmi les signatures de tous les administrateurs. Il est présent en filigrane puisque

(15) Théophile Ducroce, op. cit, p. 2. 
les arrêtés sont précédés de la formule répétitive et finalement assez formelle : « ouï le procureur général syndic » ou « le procureur général syndic entendu ». On peut dès lors le considérer, dans la ligne de l'héritage historiographique,commeune personnalité protocolaire, c'est-à-direun desélémentsmultiplesd'unrèglementadministratifnouveau,unecomposante du décor et de la mise en scène dans la décision collégiale. Le protocole étant d'autant plus important que les cadres administratifs sont récents, sans l'héritage de la pratique, sans la légitimité de l'expérience.

Ainsi, en juillet 1792, lors de l'assemblée administrative du département del'Ariège, une partie des administrateurs quittela salle pourune autre pièce dans laquelle ils prennent un certain nombre d'arrêtés sans l'aval du reste de l'assemblée. Le procureur général syndic est resté avec l'ensemble de l'assemblée et celle-ci suit son cours normalement avant d'apprendreledéroulementd'uneséance « parallèle ».Commentassurer alors la légalité des arrêtés pris en petit comité? C'est par la présence du substitut du procureur dans la pièce et par la mention de la formule « le substitutduprocureurgénéral syndicentendu »entêtedel'arrêté,queles administrateurs justifient, auprès de l'assemblée administrative puis du ministre de l'Intérieur, la légalité d'un arrêté concernant le «brûlement des titres denoblesse ${ }^{16}$. Cetteanecdote soulignel'importance du règlement dans la prise de décision administrative et la place bien particulière duprocureurquientantquereprésentantduroiestunélémentfondamental de la légalité d'une décision. Cependant, le fait qu'il soit interchangeable signifie bien ici qu'il est considéré bien plus comme une présence institutionnelle obligatoire que comme une personnalité à part entière. Oncomprend dèslorsun peu plus la faiblesseapparente des sourceset de manière corollaire l'oubli historiographique.

Toutefois, unerechercheplusapprofondiedanslesarchivespermet rapidementdefaireapparaîtredevéritablespersonnalités politiquesdans les départements. Pour cela nul besoin de surinterpréter les sources mais tout simplement de dépouiller de manière plus large et plus diversifiée les archives tant départementales que nationales. La place de procureur généralsyndicestreconnuecommefondamentaleauseindel'administration départementale et ce dès l'élection de ces hommes, souvent retrans-

(16) $A N, \mathrm{~F}^{1 \mathrm{a}}$ 404, département de l'Ariège. Procès-verbal de l'assemblée administrative du département daté du 28 juillet 1792 et lettre du procureur général syndic suppléant au ministre de I'Intérieur datée du 30 juillet 1792 : «Je fis dans cet arrêté les fonctions de procureur général syndic, comme son suppléant. L'Assemblée administrative, malgré cet arrêté, a nommé elle-même des commissaires aux mêmes fins. Elle a prétendu que nous étions sortis à cet effet pour la contredire ». 
crite comme un moment fort de l'assemblée électorale ${ }^{17}$. D'ailleurs, leur traitement n'est pas le même que celui des autres administrateurs. Ainsi, d'aprèsleregistre de départementduGers, letraitementd'unadministrateur pour un trimestre en 1792 serait de 400 livres, celui du procureur du département de 748 livres soit quasiment le double ${ }^{18}$. De même, si l'on en croit les archives organisant le département des Alpes-Maritimes le 30 mars 1793 , les procureurs généraux syndics semblent se distinguer par leur costume ou plus précisément par leur ruban et leur médaille différents de ceux des autres administrateurs ${ }^{19}$. Enfin, certains procureurs généraux jouent simplement de leur personnalité et deviennent de véritables personnages politiques locaux, à l'image de Reubell, procureur général syndic du Haut-Rhin entre mai 1790 et septembre 1792 et à propos duquel Jean-René Suratteau écrit : «II se faisait une gloire de porter une cocarde très volumineuse et faisait arborer les trois couleurs partout où il séjournait $»^{20}$. Le cas de Reubell souligne plusieurs éléments intéressants. Tout d'abord le fait que certains procureurs avaient sans nul doute une très haute opinion de leur statut et de leur rôle dans le département. Cetaspectseretrouvenotammentdanscertainsdépartementsoùleprocureur fait imprimer et afficher son réquisitoire sur des placards ou imprime ses discours pour les diffuser auprès de la population ${ }^{21}$. Le cas de Reubell illustre également la possibilité pour certains procureurs généraux de devenirdespersonnagescentrauxdudépartement,reconnuscommetels parleursconcitoyens.LecérémonialentourantledéplacementdeReubell suppose que la population elle-même identifie l'homme et sa fonction,

(17) Ibid., F'c III Charente-inférieure 3. Élections du 27 juin 1790. Un électeur fait observer « que l'on va maintenant s'occuper de la nomination du procureur général syndic; que cette nomination est de la plus haute importance et exige les précautions les plus sévères ». Selon la loi, le procureur doit être élu à la suite de tous les autres administrateurs. Cependant, on observe que certains départements réclament qu'il soit élu en premier lieu, notamment dans I'Orne (ibid., Fc III Orne, lettre des commissaires du roi au ministre de l'Intérieur le 13 juillet 1790). De plus, l'élection du procureuren derniernedoitpasforcéments'interprétercommeunesous-estimation desonimportance, même si cette réflexion serait à conduire de manière plus rigoureuse.

(18) Ibid., $F^{\text {aa }} 413$ Gers. Extrait du registre des délibérations du département du Gers (1 ${ }^{\text {er }}$ mai 1792).

(19) Ibid., $\mathrm{F}^{1 \mathrm{a}} 403$ Alpes-Maritimes. Proclamation des commissaires de la Convention nationale pour l'organisation du département des Alpes-Maritimes (30 mars 1793) : "La médaille du procureurgénéral syndic sera attachée au ruban, à la distance de deux pouces, par une tresse et deux glands de la couleur de la médaille ».

(20) Jean René Suratteau et Alain Bischoff, Jean François Reubell, I'alsacien de la Révolution française, sl, Éditions du Rhin, 1995, p. 100.

(21) C'est le cas notamment de Jard-Panvillier, procureur général syndic du département des Deux-Sèvres qui, le 29 juillet 1791, fait un rapport sur un journal antiroyaliste et le fait imprimer sur des placards affichés dans le département (AN, $F^{1 a} 436$ Deux-Sèvres). 
participant de fait à une réelle distinction sociale. Le procureur dépasse alors sa simple tâche administrative pour devenir un relais politique de la Révolution dans le département. L'affirmation des symboles de la nouvelleConstitutiondansl'ensembledudépartementestalorsunactetoutà la fois pédagogique et profondément politique.

Cesdernièresremarquesetplusglobalementlaprésencefinalement trèsnettedesprocureursgénérauxsyndicsdanslescorrespondancesavec le pouvoir central, dans les archives parlementaires et dans une moindre mesure dans les archives départementales, tendent à montrer l'importance d'une fonction qui comme toutes les institutions prend du relief à la lumière des personnalités qui l'occupent. La mise en scène qui entoure le statut de certains procureurs généraux permet de montrer la frontière trèsperméableentrepersonneladministratifetpersonnalitépolitique.En effet, le procureurgénéral syndic, malgré sa dimension protocolaire, possède un rôle départemental majeur qui se construit autour de la notion diffuse et variable d'influence.

Une institution qui se construit autour de la notion diffuse d'influence

Malgré l'obsession des constituants à vouloir limiter les prérogativesduprocureurgénéralsyndic,ceshommessemblaientdisposerponctuellement d'une certaine latitude d'action, légitimée par la volonté de faire exécuter les décisions nationales et départementales. Ainsile décret de l'Assemblée nationale du 12 juin 1790 sur la continuation de la perception des dîmes « autorise le procureurgénéral syndic à commettrequi bon lui semblera pour faire ladite perception dans les paroisses $»^{22}$. La formulation dudécretdonneicidemanière ponctuelleun pouvoirimportant au procureurgénéral syndic du département. Choisirà luiseul un collecteur d'impôt c'est à la fois influer largement sur le déroulement de la perception et jouer d'un certain pouvoir de nomination. De manière plus générale,alorsqueleprocureurpossèdeunefonctiontrèsclairementdélimitée au sein des séances du directoire, c'est-à-dire au cœur de la prise de décision, cette fonction demeure assez floue dans la mise en application de cette même décision. Il est chargé par la loi de « la suite de toutes les affaires » et de " veiller et agir pour les intérêts du département ». Cesformulationssemblentassezvaguespourpermettreàcertainesfortes

(22) Ibid., F $F^{1 a} 410$ Eure-et-Loir. 
personnalités de devenir des autorités de référence au sein du département. Ainsi, dans les interstices de la fonction, dans le flou des lois, dans les espaces d'expression laissés libres par les constituants, beaucoup de procureurs vont largement dépasser le simple rôle de « secrétaire des services administratifs » défini par Albert Soboul pour faire jouer la notion fondamentale d'influence.

C'est tout d'abord à travers l'exercice bien particulier du réquisitoire que le procureur se pose en tant qu'homme d'influence au sein de la prise de décision administrative. Il déborde alors sa simple fonction d'agent de l'exécutif pour orienter la politique du directoire. Les réquisitoires qui précèdent les débats ont souvent une forte valeur incitative, dans le contenu comme dans la formulation. Rapidement le procureur général saute le pas entre le rappel de la loi et l'exhortation à l'action en des termes parfois impératifs. À la lecture des arrêtés pris par les départements, on remarquetrèsclairementquelesadministrateurs reprennent dans leurs décisions les points soulignés par le réquisitoire. Le procureur général syndic utilise parfois des procédés très subtils pour transformer une influence diffuse en une réelle pression politique. Ainsi, le 10 septembre 1790, Barennes, procureurgénéral syndic dela Gironde prononce un réquisitoire sur les troubles dans la ville de Rions. Dans l'incapacité de prendredirectementunedécision, ilproposequeledirectoireluidonneun certain nombre de missions : dénoncer à l'accusateur public le procureur de la commune et le maire ou encore réquisitionner la garde nationale ${ }^{23}$. On distingue ici toute la subtilité du rôle du procureur, puisqu'il est à l'initiative de la décision, il est à l'exécution de cette décision, mais il ne peut être dans la prise de décision. Image même de la séparation des pouvoirs, le procureur concentre donc son influence sur la maîtrise du discours et la capacité oratoire. L'institution de procureur syndic doit doncêtre pensée au-delà dessimplestextes deloidansunepratiquequotidiennefaisantde ces hommes des référents de la politique départementale.

C'est notamment la maîtrise de la correspondance qui légitime l'influence de leurs interventions. En effet, le procureur est une sorte de pivot, de relais entre le local et le national ce qui le place au centre de la correspondance. II associe des échanges réguliers avec le pouvoir central (ministres, Assemblée nationale) et une correspondance étendue au

(23) Ibid., F $F^{1 a} 413$ Gironde. Suite à son réquisitoire, Barennes termine en ces termes: "C'est d'après ces considérations, Messieurs, que nous vous prions de prendre la délibération dont nousvousremettonsleprojetsurlebureau ».Au-delàmêmedel'influenceverbale,leprocureurgénéral syndic a ici fourni directement un projet écrit contenant toutes les décisions à prendre. 
niveau local (procureurs de district, procureurs de commune, municipalités, procureurs généraux syndics des départements limitrophes, etc.). Or, correspondre, c'est posséder l'information, ce qui revient à détenir un pouvoirdéterminantdanslaFrance desdébuts delaRévolutionfrançaise. Le procureur général syndic est un homme d'influence parce qu'il est un homme de parole et d'écriture et que ces deux compétences en font un référent, un relais entre le local et le national, entre Paris et la province. Dans les récents repères administratifs français, au cœur d'une province quicherchedenouvelles référencesterritoriales,leprocureurgénéralsyndicparticipelargementàlacohésiondudépartement.L'entretiendelacorrespondance avec les districts et les municipalités participe à la création d'une identité départementale qu'il contribue à concrétiser.

Entantqu'hommederéseau et en tantqu'individualitéidentifiable, il participe largement à la « mise en réalité » des nouveaux territoires politiques. Il incarne le département avec tout ce que cela peut réunir comme enjeux dans la France de 1790. Et même si les députés de la Constituante ontdélimitésafonctionpourévitertoutepersonnificationdudépartement enunseulhomme, ilssontlespremiersàluireconnaîtrececaractèred'incarnation. Il est ainsi souvent appelé à la barre de l'Assemblée nationale puis de la Convention pour rendre des comptes quant à l'administration du département. Le 2 avril 1792 le procureur général syndic des Bouchesdu-Rhône, Jaubert, est convoquéà la barre par les députés de l'Assemblée législative pour justifier la conduite des administrateurs du département pendant les troubles de la ville d'Arles. Il lui est réclamé de faire un exposé de la situation dans le département et des prises de décision du directoire dedépartement.Ilapparaîtdanscettesituationdavantagecommesolidaire de la politique départementale que comme représentant de l'exécutif. II se positionne lui-même dans l'administration quand il conclut son long exposé en ces termes: «Vous voyez messieurs que nous ne cessions de nousoccuperdecettevillelorsquenosfonctionsontétéinterrompues $»^{24}$. Ainsi, même le pouvoir central navigue entre la volonté de limiter son influence dans le département et l'utilisation de son statut comme celui d'un porte-parole de ce même département. On retrouve donc cette tension propre à l'institution, cet entre-deux où certaines personnalités vont réussir à s'affirmer au gré des opportunités et des circonstances.

(24) AP, tome 41, séance du 2 avril 1792. L'appel des procureurs généraux à la barre de I'Assemblée sefait plus fréquent à partir de 1792, lorsque les tensionss'affirment dans certains départements français. La convocation du procureur comme représentant de son département n'est donc pas un principe établi par les constituants à la naissance de l'institution mais bien plus une réaction pragmatique face à une situation délicate. 
Le rôle des personnalités et des circonstances : l'exemple des départements frontaliers

Le cas des départements frontaliers questionne les fondements de l'institution de manière aiguë, car ces territoires cumulent un fort problème identitaire et un contexte de tensions, voire de conflits armés à partir de 1792. Dans les négociations avec les puissances limitrophes, dans la circulation des informations et des rumeurs concernant les dangers que court la patrie, les procureurs généraux syndics apparaissent souvent commedesinterlocuteurs.Cesontdesconditionsquipermettentàcertains de donner une épaisseur supplémentaire à leur action en se fondant sur des circonstances extraordinaires. Ainsi, dès 1790, Poutet, alors procureur général syndic de la Moselle, informe le ministre de l'Intérieur du contenu d'unelettreprovenant du ministre de Deux-Ponts et concernant les dispositions de l'ennemi. II se permet même d'« inciter » le ministre de l'Intérieur à contacter le ministre de la Guerre. Poutet est donc bien un relais de l'information, celui qui détient les connaissances au sein du département. Cetteposition le poseen interlocuteurprivilégiédela politiquedugouvernement, voire en conseiller local du ministre de l'Intérieur.

De même, Llucia, le procureur général syndic des PyrénéesOrientales reçoit en 1790 une lettre d'un Français d'Espagne lui exposantlesdangersdel'armementespagnol.Cemystérieuxanonymeconclut en ces termes: « Je ne le confie qu'à vous monsieur, pour que vous en fassiez l'usage qui vous paraîtra le plus convenable $»^{25}$. Cette lettre nous permetdesoulignerplusieurscaractéristiquespropresàlafonctiondeprocureur.Tout d'abord Llucia semble intégré dans un réseau relationnel qui dépasse les frontières nationales, lui assurant ainsi des informations bien plus larges que celles émanant de son seul département. Et d'autre part, ilsembleraitqu'ens'adressantàlui,soncorrespondantattendedesconséquences directes, des décisions politiquesetsurtoutuntransfertdel'information aux détenteurs de la capacité d'agir. La place de secrétaire de la loge de la Sociabilité, occupée par Llucia avant la Révolution, peut être un élément d'explication de ce réseau régional et de l'anonymat de son informateur, qualifié par Llucia de « Français très connu résident depuis longtemps à Barcelone ». Ces quelques remarques laissent poindre une desproblématiquescentrales detouteétudesurles procureursgénéraux

(25) AN, $F^{1 a} 429$ Pyrénées-Orientales, Lettre du procureur général syndic au ministre de I'Intérieur (12 août 1790). 
syndics, à savoir la question des réseaux de sociabilité et des sphères de notabilité. Le procureur s'inscrit à un point nodal du rapport entre Paris et la province, parce qu'il associe une forte implantation locale et une possible communication avec le pouvoir central. Llucia agit d'ailleurs dans ce sens puisqu'il transfère cette lettre au ministre de l'Intérieur le 12 août 1790. De plus, en tant que détenteur d'informations fondamentales, Llucia se sent en partie responsable de la protection de son département et prend l'initiative de prévenir les municipalités frontalières du dangerpotentiel.Orlàencoretoutel'ambiguïtédel'institutionnousapparaît puisque le ministre de l'Intérieur, après avoir remercié le procureur pour les informations fournies, lui reproche de s'être adressédirectement aux municipalités, l'accusant de vouloir semer la terreur sur de simples spéculations. Par ce rappel à l'ordre, le ministre cantonne Llucia à son rôle : il est un relais de l'information mais en aucun cas il ne peut prendre de décision en son nom sans outrepasser ses attributions. Pour autant, la guerre et la difficulté de la situation dans certaines régions donneront au procureur général syndic une place de plus en plus importante.

C'estnotammentle cas dans les départements périphériques à partir de 1792, à l'image de Reubell dans le Haut-Rhin ou encore de Gossin dans la Meuse. Ce dernier apparaît, dès sa prise de fonction, comme un procureurgénéralsyndicparticulièrementinfluentsurlapolitiquedépartementale. Dès 1791 ses réquisitoires sont retranscrits en tête des arrêtés du directoire et orientent clairement les décisions prises par le département dans tous les domaines (gestion des récoltes, prêtres réfractaires, impôts, subsistances, justice de paix, volontaires nationaux, brigandage, production de vin, etc.). Une telle personnalité montre la latitude d'action de certains procureurs qui, sans sortir de leur rôle, prennent une réelle ampleur au niveau local, a fortiori en période de conflit. En effet, dès 1792, Gossin est particulièrement actif dans les affaires delaguerreau point de devenir l'interlocuteur de référence pour les autorités prussiennes qui s'adressent directement à lui et le chargent par exemple, d'organiser le ravitaillement de leur armée dans le département ${ }^{26}$. Le poids des circonstances justifie en partie la montée en puissance du personnage. Sa fin tragique illustre

(26) Ibid., $\mathrm{F}^{1 \mathrm{a}} 423$ Meuse. Le grand conseil de guerre du roi de Prusse s'adresse ainsià Gossin le 5 septembre 1792 : «D'après les représentations que vous m'avez faites, en date d'aujourd'hui, j'ai eu soin qu'on donne des ordres, pour qu'aucun détachement prussien n'occupe Bar. Je trouve cettecondescendance pour le siège du département très juste afin de ne pas obérerl'administration par une charge qui pourrait causer une stagnation dans ses pouvoirs et nuireà la bonne cause ». Il est doncl'interlocuteurprivilégié,ledéfenseurdudépartementauprèsdesautoritésprussiennes, brefen quelque sorte le « représentant » du département. 
encore une fois la problématique de la fonction. En effet, Gossin est guillotiné en juillet 1794 après s'être caché pendant des mois à la suite des accusations portées contre lui. C'est notamment le fait d'avoir obéi aux ordres de Brunswick lors de l'invasion de Verdun qui achève de le rendre suspect aux yeux de la Convention. Là encore, la limite semble bien floue dans la tête de certains procureurs entre le rôle de relais institutionnel et la prise d'initiative politique. Si le poids des circonstances dans les départements frontaliersjoueun rôlefondamental danslapratiquedel'institution,lapersonnalitéde ces hommes demeurela cléd'interprétation principale. Dans la Meuse, la guerre a permis à Gossin de prendre une dimension politique importante, au contraire dans le Nord, dès avril 1792, le conflit fait disparaîtreleprocureurgénéralsyndicdelacorrespondanceaveclepouvoircentral. Un conseil exécutif provisoire devient l'organe de référence dans la correspondanceaveclegouvernement ${ }^{27}$.Lemanqued'informationconcernant la carrière et la biographie du deuxième procureur, Delval Lagache, permet de supposer qu'il ne possédait pas l'épaisseur d'une personnalité politique.Riend'automatiquedoncdansuneanalysenationaledecetteinstitution mais un travail à faire sur la nuance, l'initiative et la personnalité de ces hommes enserrés dans les tensions de leur propre institution. Le procureurgénéralsyndicestàlafoisuneprésenceadministrativeformelle etunpersonnageréférentauniveaududépartement.C'estsansdoutecette tension qui l'a fait apparaître souvent comme un homme de conciliation, comme un modéré sans pouvoir et donc sans intérêt. Maisc'est aussicette tension qui porteen germel'intérêt du personnage puisqu'elle permetun éclairage particulier sur le devenir politique de ces hommes. En effet, les problématiquescoupléesd'influenceetdeconciliation prennenttoutleur sens au xIx siècle dans la succession des régimes et des frontières politiques. La prosopographie peut alors permettre d'établir un lien entre la particularitédel'institution deprocureurgénéralsyndicetl'apprentissage d'un certain savoir-faire politique et administratif.

Une redécouverte de l'institution par l'approche prosopographique

La prosopographie a prouvé depuis plusieurs décennies sa pertinence dans l'analyse d'un corps social et dans la réflexion sur la notion de notabilité. Concernant les procureurs généraux syndics, elle devient

(27) Ibid., F 426 Nord. Le procureur général syndic est de Warenghien jusqu'en octobre 1792, puis Delval Lagache sur lequel on a d'ailleurs peu de renseignements précis. 
aussi un outil méthodologique de réhabilitation historiographique. « La reconstitutionmonographiquedescarrièresetdesparentéspuisleurmise ensérie ${ }^{28}$ permettentd'envisagerdemanièrecomplémentairel'étudedes individusetdel'institution.Ainsiunebiographiecollectivedesprocureurs généraux syndics participe à une réflexion plus large sur la formation d'une nouvellegénération politique pendantla Révolution etcollaboreà unevéritablesociographiedel'État.Ladémarcheprosopographiquepermet une approche diachronique, elle n'isole pas la Révolution française comme objet d'étude en tant que tel mais elle la replace dans un avant et un après par la continuité des destins individuels.

Labiographiecollectivedeceshommesillustredesthèses déjà soulignées par l'historiographie de la Révolution à savoir le maintien d'une partie du personnel administratif de l'Ancien Régime qui cohabite avec unemassed'hominesnoviaccédantauxresponsabilitésgrâceauxbouleversements révolutionnaires. L'analyse de l'institution met donc en lumière à la fois la permanence d'un certain savoir-faire administratif mais aussi un véritableapprentissage du politique. Orc'est justement cet apprentissagedupolitiquequipeutguiderlequestionnement prosopographique. Être procureurgénéral syndicau début dela Révolution correspondà une manière particulière d'apprendre la pratique administrative et politique. On peut doncse poser la question suivante :quel est le lien entrel'originalité de l'institution de procureur général syndic et les destins individuels de ces hommes? En d'autres termes, est-ce que le fait d'avoir été procureur général syndic est un élément explicatif, voire central de l'évolution d'une carrière? Ce qui est d'autant plus pertinent dans une période de changementspolitiquespermanents.Enrecherchantunsystèmepolitique stable pendant la majorité du xix $x^{e}$ siècle, la France se cherche également une classe dirigeante encadrant la société à tous les niveaux. Les acteurs de la Révolution naviguent alors souvent entre la revendication de leur savoir-faire administratif et la justification de leurs choix politiques. Éliminésparlegouvernementrévolutionnaire,lesprocureursgénérauxsyndics sont pour la plupart débarrassés du lourd fardeau « terroriste», ce qui les place dans une position souvent favorable à partir de Thermidor pour entamer une carrière administrative, politique ou judiciaire.

L'étude de l'institution et de la pratique de ces hommes au sein de l'administrationdépartementalepermetdedégageruncertainnombrede

(28) Christophe Charle, Jean Nagle, Marc Perrichet, Denis Woronoff et Michel Richard, Prosopographie des élites françaises, Paris, Guide de recherche du CNRS, 1979. 
caractéristiques propres àla fonction de procureurgénéral syndic.Leprocureurgénéral syndic est une personnalité du consensus associant esprit de conciliation et réseau relationnel. C'est également un homme attaché à l'autorité de la loi et au respect de la hiérarchie administrative adoptant souventuncomportementquel'on peuttaxerdelégaliste.Il possèdedans la plupart des cas un réel savoir-faire administratif et juridique grâce à son métier d'origine (homme de loi, administrateur provincial...) mais aussi par son acculturation politique au sein du creuset de l'administration départementale. Enfin, le procureur général syndic est attachéà une certaine distinction sociale liée à son statut de notable mais aussi à sa place au sein de l'administration : il est un individu identifiable face à une instance collégiale. Consensus, légalisme, savoir-faire et distinction sont des caractéristiques largement attachées à l'institution elle-même mais elles sont aussi les éléments centraux de la compréhension d'une carrière. Ces qualités ne s'associent pas à n'importe quel itinéraire individuel, elles sont exploitées dans des parcours bien particuliers. En mettant en évidence le lien entre l'institution et les carrières, il devient possible de réhabiliter l'institution des procureurs tant par la réalité de leur action dans les départements que parl'importance de leur pratique administrative lors de la création de la nouvelle classe dirigeante.

Larecherchedesinformationsbiographiqueslesconcernantapermis d'établir une liste de 199 procureurs généraux syndics dont 157 possèdent une notice individuelle ${ }^{29}$. Les différences sonténormes entre une notice de quelqueslignesetleslonguesbiographiesquiretracentlaviedesprocureurs aux destinées les plus remarquables (Beugnot, Boissy d'Anglas...). C'est à la fois l'atout et le défaut de toute recherche prosopographique puisque letraitementquantitatifmetsurlemêmeplandespersonnalitésderayonnement très divers. Dans le même temps, cela permet de redonner une place dansl'histoireà des hommes dont on connaît pourtant peu de choses, simplement en les incluant dans le traitement statistique. La mise en série des noticesindividuellesamèneàdégagerquatregrandesproblématiques.Tout d'abord le recrutement des procureurs généraux syndics par un suffrage

(29) Nous nous devons ici de faire, de nouveau, référence au travail universitaire de Gilbert SCHNEIDER, op. cit. Nous avons en effet repris l'ensemble de cette recherche pour amorcer notre propretravailet notammentlesnombreuses notices biographiquesélaborées parl'auteur. La listede 199 procureursest prochedel'exhaustivitémaisneprenden comptequeleshommesélusentre 1790 et 1793. Elle exclut donc les procureurs généraux syndics de l'an III ainsi que les procureurs généraux nommés par les représentants du peuple en mission à partir de l'automne 1793 pour remplacer les administrateurs soupçonnés et destitués. 
censitaire qui définit in fine le portrait de la notabilité révolutionnaire ${ }^{30}$ et qui pose cette question à la fois simple et fondamentale :qui est procureur général syndic? La seconde problématique regroupe les itinéraires et les attitudes pendant la Révolution. La question n'étant plus qui est procureur mais plutôt quels procureurs sont-ils? Cet axe de réflexion posela question dela participationau processus révolutionnaireen interrogeantl'incompatibilité structurelle entre l'institution et le principe révolutionnaire ${ }^{31}$. Le fait que 47 procureurs généraux soient suspectés en l'an II (dont 29 étaient en fonction et furent destitués) illustre en effet les impasses de cette fonction, imbriquée dans les réseaux de fidélitélocaux, face aux tensions internes de la société révolutionnaire et surtout face au fédéralisme ${ }^{32}$. Ces premières conclusionsébauchéespermettentàlaprosopographied'apporterdeséléments de réponse sur l'événement révolutionnaire lui-même mais aussi à toute une réflexion concernant la construction de l'État en France. Une troisième problématique s'articule autour des carrières proprement dites lors des régimes politiques successifs prolongeant la Révolution. Après la destruction des ordres anciens, après les bouleversements de la décennie révolutionnaire, la France a construit successivement les bases sociales de sanouvellesociété.Lesanciensprocureursgénérauxparticipentdecelarge mouvement, intégrant les « masses de granit » et les sphères de notabilité par la promotion méritocratique et la réutilisation d'un savoir-faire solide. Enfin, toute étude prosopographique se doit de considérer les stratégies de promotion sociale. Au-delà des carrières politiques, administratives et judiciaires, les procureurs généraux participent souventà lagrande « redistribution » de la richesse et de la reconnaissance sociale qui suit la Révolution à travers le choix d'une épouse ou l'achat d'un bien national. Pour autant, l'idée de « stratégie » individuellenedoitjamais cacher auxyeux du chercheur les véritables relations affectueuses, les amitiés sincères ou tout

(30) Lesuffrage restreint parl'impôt permet deconsidérerl'élection comme une reconnaissance par les pairs. L'absence de candidature aboutit souvent à la promotion de personnalités locales ou d'un « patriciat de renommée » selon l'expression de Saint-Just reconnaissant officiellement l'influence préexistante d'hommes reconnus par tous.

(31) Parmiles 157 procureurspossédantdesnoticeson peutcompter23élusauxÉtatsgénéraux (dont 5 suppléants), 33 élus à la Législative (dont 4 suppléants), 38 élus à la Convention (dont 10 suppléants). Ce simple décompte permet déjà de penser la fonction de procureur général syndic dans le processus révolutionnaire dans son ensemble. Les procureurs furent des acteurs révolutionnaires au sens large du terme.

(32) C'est cette incompatibilité entre la France révolutionnaire et son administration qui fait dire à Saint-Just en octobre 1793 : «Un peuple n'a qu'un ennemi dangereux, c'est son gouvernement ». Rapport du 10 octobre 1793, CEuvres de Saint-Just, Paris, Fasquelles, 1908, tome 2, p. 75-76. Il faut entendre ici gouvernement dans le sens d'administration. 
Les variables à saisir dans le traitement statistique

\begin{tabular}{|c|c|c|c|}
\hline Le recrutement & $\begin{array}{c}\text { Les attitudes } \\
\text { pendant la Révo- } \\
\text { lution }\end{array}$ & $\begin{array}{l}\text { Les carrières } \\
\text { postérieures }\end{array}$ & $\begin{array}{l}\text { Les stratégies de } \\
\text { promotion sociale }\end{array}$ \\
\hline $\begin{array}{l}\text { La notabilité } \\
\text { prérévolutionnaire: } \\
\text { - Ordre } \\
\text { - Charges sous } \\
\text { l'Ancien Régime } \\
\text { - Métier } \\
\text { - Mandats locaux } \\
\text { avant d'être pro- } \\
\text { cureur } \\
\text { - Profession dupère }\end{array}$ & $\begin{array}{l}\frac{\text { Participation active }}{\text { auprocessus révolu- }} \\
\text { tionnaire: } \\
\text { - Participation aux } \\
\text { assemblées. } \\
\text { - Envoi en mission } \\
\text { - Participation aux } \\
\text { comités. } \\
\text { - Membres des } \\
\text { - clubs } \\
\text { - Publications }\end{array}$ & $\begin{array}{l}\text { Participation } \\
\text { politique: } \\
\text { - Conseil des Cinq } \\
\text { Cents } \\
\text { - Conseil des } \\
\quad \text { Anciens } \\
\text { - Corps législatif } \\
\text { - Tribunat } \\
\text { - Sénat } \\
\text { - Chambre... }\end{array}$ & $\begin{array}{l}\text { Promotion } \\
\text { économique: } \\
\text { - Inscription sur les } \\
\text { listes de notabi- } \\
\text { lités } \\
\text { - Inventaire après } \\
\text { décès. } \\
\text { - Achats de biens } \\
\text { nationaux. }\end{array}$ \\
\hline \multirow[t]{3}{*}{$\begin{array}{l}\text { Héritage culturel } \\
\text { et intellectuel: } \\
\text { - Lieu des Études } \\
\text { - Franc-maçonnerie } \\
\text { - Publication avant } \\
\text { la Révolution } \\
\text { - Membreoucorres- } \\
\text { pondant d'Aca- } \\
\text { démie }\end{array}$} & $\begin{array}{l}\text { Suspicion et contre } \\
\text { révolution: } \\
\text { - Émigration } \\
\text { - Suspects } \\
\text { - Arrestation } \\
\text { - Exécution }\end{array}$ & $\begin{array}{l}\frac{\text { Attitude et }}{\text { opinions: }} \\
\text { - Attitude au } 18 \\
\text { brumaire } \\
\text { - Vote de la } \\
\text { déchéance de } \\
\text { l'Empereur en } 1814 \\
\text { - Participation aux } \\
\text { Cent-Jours. } \\
\text { - Attitude à la } \\
\text { Restauration. }\end{array}$ & $\begin{array}{l}\text { Promotion sociale } \\
\text { - Épouse } \\
\text { - Dot } \\
\text { - Profession dupère } \\
\text { de l'épouse. } \\
\text { - Profession des } \\
\text { enfants. }\end{array}$ \\
\hline & $\begin{array}{l}\text { Opinions politiques } \\
\text { - Vote au procès } \\
\text { du roi. } \\
\text { - Attitudele } 10 \text { août } \\
1792 . \\
\text { - Attitude le } 31 \text { mai } \\
1793 \\
\text { - Attitude au } 9 \\
\text { thermidor an II }\end{array}$ & $\begin{array}{l}\text { Carrières locales: } \\
\text { - Préfectures } \\
\text { - Fonctions judi- } \\
\text { ciaires } \\
\text { - Administration } \\
\text { locale }\end{array}$ & $\begin{array}{l}\frac{\text { Les réseaux }}{\text { relationnels: }} \\
\text { - Société de pensée } \\
\text { - Témoins au } \\
\text { mariage, à la suc- } \\
\text { cession } \\
\text { - Correspondance }\end{array}$ \\
\hline & & \begin{tabular}{|l}
$\frac{\text { Reconnaissance }}{\text { sociale, titre }}$ \\
honorifique \\
- Légion d'honneur \\
- Noblesse d'Empire \\
- Pairie \\
- Académies, \\
Institut
\end{tabular} & \\
\hline
\end{tabular}


simplementlesréellescompétencesprofessionnelles ${ }^{33}$.Autrementdit,place doit être faite à l'individu, au « beurre sur le pain » d'après l'expression de Louis Bergeron et de Guy Chaussinand Nogaret ${ }^{34}$.

Bienévidemment,letraitementstatistiquedesnoticesindividuelles permet de faire émerger une sorte de portrait type du procureur général syndic. Membre du troisième ordre, il est homme de loi sous l'Ancien Régime (128 membres de professions juridiques dont 102 avocats), il participe au moins à une assemblée révolutionnaire entre 1789 et 1795 (58procureurssontéluscommetitulaireousuppléant)etpoursuitavecun mandat de député sous le Directoire (54 membres du Conseil des CinqCents ou du Conseil des Anciens). Alors que son investissement dans la chose publique est souvent reconnu par un titre honorifique au $x \mathrm{x}^{\mathrm{e}}$ siècle (Légion d'honneur, noblesse d'Empire, etc.), il termine sa carrière dans une haute fonction judiciaire au cœur de son département d'origine (31 procureurs auront une haute responsabilité judiciaire sous le Consulat etl'Empire).Ceprocureurgénéral syndic stéréotypéa souvent connuune réelle promotion économique au cours de sa vie et voit son nom inscrit surleslistesdenotabilités départementalesduxixe siècle.Inévitablement, au fur et à mesure de l'analyse diachronique les chiffres semblent moins éloquents. Mais il faut toujours considérer la diminution importante de l'effectif concerné puisqu'en 1800 plusieurs procureurs sont morts ou ont complètement quitté la vie publique ${ }^{35}$. Si la construction dece rapide " portrait-robot »peutsemblerune démarcheinévitable, ellen'esten rien suffisante tant du point de vue de la méthode que del'intérêt historiqueà proprement parler.

L'ambition d'une étude prosopographique ne peut pas être la définition d'un portrait figé mais bien au contraire la mise en évidence d'une

(33) Yves Marie Bercé dans la préface de l'ouvrage de Nicole Gotterl, Claude Petiet, ministre de la Guerre, intendant de la grande armée et ses fils Alexandre, Auguste et Sylvain, Paris, Éditions SPM, 1999, écrit ainsi : « Les découpages chronologiques dictés par les grands événements politiques donnent inévitablement une image très fausse de la réalité de la vie ; ils ignorent des faits simples, tels que la volonté de survivre en dépit des malheurs politiques, tels que le déroulement des existencesdesunset desautresenjambantles règneset les révolutions; ilsnesavent pas tenircompte du maintien des solidarités et des cousinages, des jalousies et des affections, ils oublient les innombrables et modestes histoires individuelles faites de bonheur ou de malheurs obscurs ».

(34) Louis Bergeron, Guy Chaussinand Nogaret, Les grands notables du second Empire, Paris, Éditions du CNRS, 1978. Dans la préface de cet ouvrage les deux auteurs se prémunissent contre les défauts d'un traitement qui serait trop statistique et fixent leurs objectifs en ces termes: «Au-delàdumasquesavant, retrouverlevisagequotidienetlessingularités régionales, et desphysionomies multiples faire jaillir les traits communs ».

(35) Nous nous référons ici aux informations détenues pour l'instant. Nous possédons la date de décès pour 110 procureurs et sur cet échantillon, 23 sont morts avant 1800 . 
figure complexe, à l'image des champs de forces multiples de la société post-révolutionnaire. L'étude quantitative de ces itinéraires individuels et la confrontation avec les personnalités distinctes qui en émergent permettent d'envisager le procureur général syndic comme une figure polymorphe. Il incarne finalement la rencontre entre les espérances - voire les utopies - constitutionnelles de 1789 et une réalité politique instable et complexe qui s'impose dès 1792. L'analyse des carrières permet donc d'identifier plusieurs figures. Ces figures, au-delà de la simple étude de l'institution, sont finalement des personnages de la vie publique du $\mathrm{xIX}^{\mathrm{e}}$ siècle, des profils d'hommes rencontrés sur la scène politique, dans les salons, dans les villes de province, dans la littérature de l'époque. Nouspouvonsainsidistinguerplusieursfigures :lafiguredelagirouette ${ }^{36}$, la figure du serviteur de l'État promu par le mérite, la figure de l'homme deréseau, celledunotablelocal ou encorecelledu technicien administratif alliant expérience et savoir-faire. Les différentes facettes du procureur général syndic se croisent et se décroisent au gré des circonstances et des personnalités. Elles dessinent plusieurs portraits, plusieurs profils dans lesquels on retrouve les caractéristiques propres à leur apprentissage du politique : le consensus, le légalisme, le savoir-faire et la distinction. La prosopographienese conçoit doncpasicicommeunefin en soimais bien commeunoutilpourdémontrerquelesparticularitésinstitutionnellesde lafonction de procureurgénéral syndic ont eu un rôle dans les particularités individuelles des destins politiques ${ }^{37}$.

Au sein de l'administration départementale le procureur général syndic symbolise la loi et le respect de la hiérarchie administrative. Il est

(36) On peut se référer aux nombreux ouvrages sur la figure de la girouette publiés dans la première moitié du XIX ${ }^{\mathrm{e}}$ : Alexis EYMERY, Dictionnaire des girouettes ou nos contemporains peints par eux-mêmes, Paris, 1815, ou encorel'Almanach des girouettes ou nomenclature de personnages marquants dont la versatilité d'opinion donne droit à l'Ordre de la girouette, Paris, 1815. La réflexion historique sur la figure de la girouette a été récemment menée par Pierre SERNA dans La République des girouettes, 1789-1815 et au-delà. Une anomalie politique : la France de l'extrême centre, Seyssel, Champ Vallon, 2005. Ouvrage dans lequel il apporte une réflexion sur cette France de l'extrême centre où il analyse « les parcours biographiques comme autant de modes de survies dictées par le pragmatisme » (p. 18).

(37) Bienévidemment, il faudra considérer de manière rigoureusel'influence delafonction deprocureurdanslacarrière.Selonlespersonnescetteexpérienceauraétéinauguraleouaucontraire aboutissement. De même un hommeayantété procureur général syndic pendant quelques mois en 1793 alors que l'institution est déjà largement décriée n'aura pas vécu le même apprentissage que le procureurélu en juin 1790 pour deuxannées de travail assidu et reconnu par tous. End'autres termes l'expérience l'aura considérablement moins marquée et donc moins orientée quant à sa future carrière. Toute étude statistique doit donc intégrer un facteur relatif fondamental. 
donclegarant dela légalitéet rappelleau directoire, commeà l'ensemble de la population, que chaque département appartient à un tout indivisible, encadré par une même Constitution. Ainsi, s'il incarne une identité départementale nouvelle, il est également le lien essentiel avec le cadre national. Or comment incarner l'exécutif après la fuite du roi avortée à Varennes? Comment se référer à la légalité après la prise des Tuileries le 10 août 1792 ? Comment assurer le respect de la loi au cœur du processus révolutionnaire?

Dèsl'automne de 1792, les procureurs générauxsyndicssontmoins présents dans la correspondance avec le ministère de l'Intérieur comme dans la politique départementale. La chute de la monarchie constitutionnelle ne leur donne qu'un sursis puisque le fondement même de l'institution est ébranlé. En 1793, les procureurs sont souvent réduits au rôle d'exécutants, subordonnésauxreprésentants du peupleen missionetaux commissaires de l'exécutif envoyés dans les départements. Les derniers mois signent déjà la fin d'une institution condamnée par la Révolution. Le problèmeestsimple :lesprocureursgénérauxsyndicsnesontpasdesrévolutionnaires et ne peuvent pas l'être puisqu'ils ne sont jamais dans la prise de décision. Pour certains, la créativité politique se vit alors au cœur de la contre-révolution, lorsqu'ilsabandonnentlaréférenceàunelégaliténationale sans cesse redéfinie pour se référer à la seule légitimité locale. Pour d'autres,lescassuresrévolutionnairesapparaissentcommedescatalyseurs dans la construction d'une carrière plus ambitieuse. Mais pour la majorité, au-delà du processus révolutionnaire, la question de la légalité reste au cœur de leur parcours dans les destins politiques comme dans les carrières judiciaires, dans les promotions nationales comme dans les implantations locales. Tout travail sur ces hommes doit donc dépasser l'échec de 1793 pour envisager l'institution à travers les questions qu'elle soulève. Le procureurgénéral syndics'inscritfinalement au cœur de plusieurs problématiquesfondamentales.llestplacéàlafrontièreentretechnicitéadministrativeetresponsabilitépolitique,entrecarrièrelocaleetcarrièrenationale et donc de manière corollaire entre la notabilité et l'élite.

Gaid ANDRO GHRis - Université de Rouen

17 rue Auguste-Blanqui 35000 Rennes gaid.andro@voila.fr 